\title{
Development of a sustainable construction system for temporary structures
}

\author{
N. De Temmerman \& L. Alegria Mira \\ Department of Architectural Engineering, \\ Vrije Universiteit Brussel, Belgium
}

\begin{abstract}
Non-static structures that can adapt their shape, or which can be quickly and easily deployed to perform their architectural function and removed afterwards without damaging sensitive sites, are well-equipped to meet the demands of a rapidly changing society which embraces the concept of sustainable design.

Generally, mobile deployable structures are capable of transforming from a small, closed or stowed configuration to a much larger, open or deployed configuration. In architecture, the main applications are temporary lightweight structures such as emergency shelters or exhibition and recreational structures. For these purposes deployable scissor structures are most effective. They consist of beam elements connected by pivot joints, allowing them to be folded into a compact bundle for storage or transport. Subsequently, they are deployed, demonstrating a huge volume expansion. This process can be reversed, allowing reuse.

In this paper an innovative kit-of-parts system is developed for deployable scissor structures. A new multi-configurational Universal Scissor Component (USC) is geometrically designed to create scissor structures in a generic way.

The designed USC - single and unique - is used to compose different configurations. The structures can be disassembled and the USC can then be reconfigured, enabling reuse (cfr. the toy construction system Meccano). The component's uniformity favours mass-production, implying a cost benefit. Moreover, the diversity in possible structural forms invites multiple uses.
\end{abstract}

Keywords: transformable structures, temporary structures, deployable structures, scissor structures. 


\section{Introduction}

\subsection{Transformable structures}

4D design (four-dimensional design) entails a design attitude in which time is explicitly included from the earliest stages of conception. So, besides the 3dimensional space, the fourth dimension becomes a determining design parameter. The structure is transformable over time and can be described as being relocatable, reuseable, demountable; the building components can be reconfigurable, removeable, replaceable, etc. Temporary structures which have this transformational capacity, and are lightweight or easily removeable, have a lower impact on the site which makes them ecologically favourable.

Generally, sustainable design can interact on one or more of three levels: (1) material (e.g. recycling, up-or downcycling), (2) component (e.g. reuse, reconfiguration), (3) structure (e.g. retrofitting, flexible renovation with infill).

In this research, focusing on the component level, the methods used for implementing transformation are kit-of-parts systems and structural mechanisms.

\subsubsection{Kit-of-parts systems}

This concept relies on the philosophy of designing and building like a 'Meccano' system with dry, reversible connections allowing a gradual transformation of the structure over time (Figure 1). Only a few components are used as the basic building blocks, but with the possibility to compose a myriad of configurations (cfr. Lego system).

A generative dimensioning system is used as an underlying geometric grid and guarantees that existing and future components will be compatible with the system. This enables the design to meet changing needs of a dynamic society, gradually over a certain period in time.
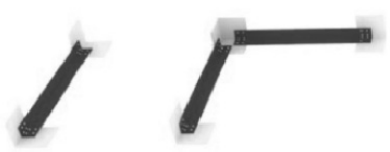

Figure 1:
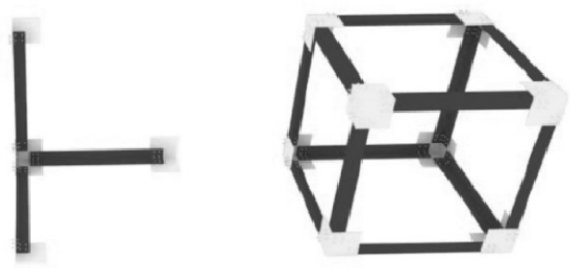

Example of a kit-of-parts system (C) Michael Lefeber).

\subsubsection{Structural mechanisms}

With the introduction of a mechanism into a structural system, it is equipped with the ability to transform from a small, closed or stowed configuration to a much larger, open or deployed configuration (Figure 2). The obtained structures are generally referred to as deployable structures, which can transform instantaneously [1].

Deployable structures are characterized by their dual functionality as loadbearing structures or mechanisms. As load-bearing structures they transfer live 


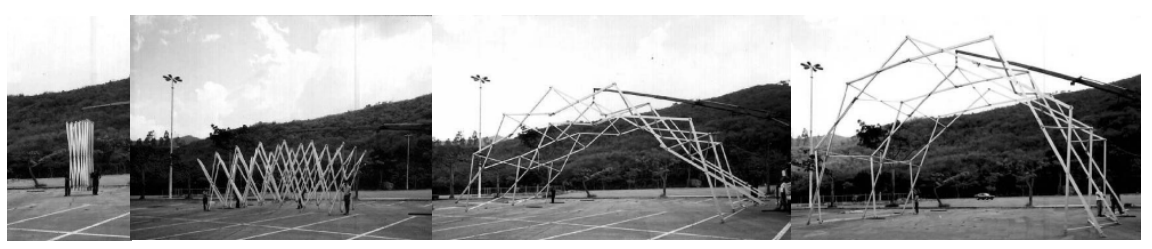

Figure 2: $\quad$ Example of a deployable scissor structure (C Grupo Estran).

and dead loads. As mechanisms they provide the reversible alteration of their form, making them adaptable to altered boundary conditions [2].

Although the research subject of deployable structures is relatively young, the principle of transformable objects and spaces has been applied throughout history (the Mongolian yurt, the pantographic weightlifting crane of Leonardo da Vinci...). Nowadays, the main application areas are the aerospace industry, requiring highly compactable, lightweight payload (solar arrays), and architecture, requiring either fixed-location retractable roofs for sports arenas (Wimbledon) or mobile, lightweight temporary shelters (emergency tents and recreational structures) [3].

Generally, mobile deployable structures consist of a weather protecting cladding supported by some form of erectable structure, which is capable of easily being moved in the course of normal use and can be assembled at high speed, on unprepared sites, for plural human activities. For this purpose, scissor structures are most effective: besides being transportable, they have the great advantage of speed and ease of erection and dismantling, while offering a huge volume expansion [4].

\subsection{Sustainable transformable construction system: Meccano and mechanism}

In this paper, an innovative construction system for deployable scissor structures is developed based on two levels: (i) the reuse of a structure and (ii) the possible reconfiguration of its constitutive components. While current designs of scissor systems give an ad hoc solution, this research provides a methodology for designing a transformable system resulting in generic scissor structures.

The scissor component, the Universal Scissor Component (USC), is single and unique and designed to compose a vast number of different architectural configurations. So, on one hand the developed scissor structures can be transported and deployed on site making the structure reusable, and on the other hand the USC can be reconfigured and reused in different geometric forms. In this way, the Universal Scissor Component combines the two aspects kit-of-parts and structural mechanism, resulting in a sustainable construction system for temporary applications which can easily meet changing requirements. 


\section{Scissor units}

Scissor units, also called scissor-like elements (SLEs) consist of two beams connected through a revolute joint, the intermediate hinge, allowing a relative rotation, but at the same time introducing bending moments in the beams. By connecting such SLEs at their end nodes by hinges, a linkage is formed, which can be transformed from a compact bundle of elements to a fully deployed configuration. If linkages are connected to each other according to a three dimensional grid, a deployable scissor mechanism is formed which can be used for architectural purposes (Figure 2). Finally, by adding constraints, the mechanism goes from the deployment phase to the service phase, in which it can bear loads.

Depending on the location of the intermediate joint and the shape of the beams, three main unit types can be distinguished: translational, polar and angulated units (Figures 3, 4 and 5).

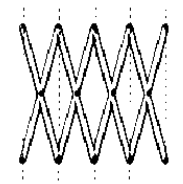

Figure 3: $\quad$ Translational linkage [5].
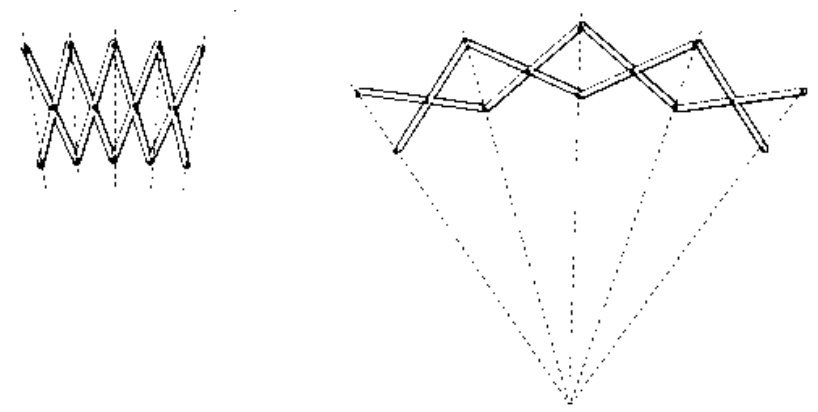

Figure 4: Scissor mechanism of polar units [5].
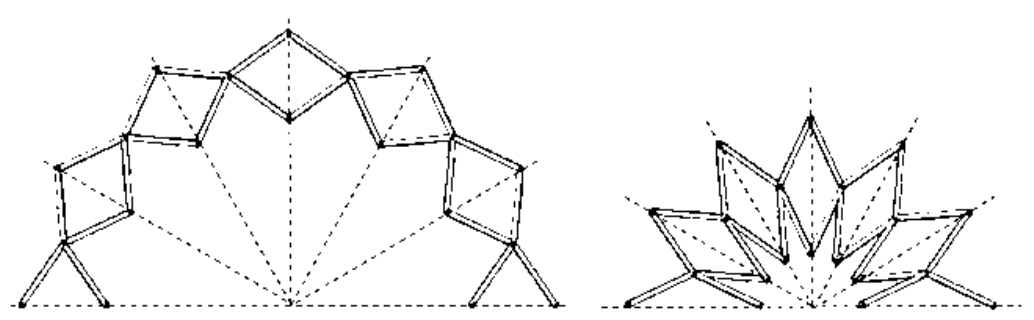

Figure 5: A radially deployable linkage consisting of angulated scissors with kinked beams [5]. 


\section{Development of a Universal Scissor Component (USC)}

\subsection{Configurations of the structures}

To determine the geometric dimensions of the USC, a closer look has to be taken which configurations of structures to consider. Deployable structures with a function of a temporary shelter are expected to enclose a three-dimensional space $[6,7]$.

\subsubsection{Barrel vaults}

To perform an architectural function (providing weather protection) a barrel vault is a simple, but effective typology. Barrel vaults or cylindrical grids are monoclastic shapes. They can be obtained by curving one direction of an orthogonal two-way grid.

Using polar units is an effective way of introducing single curvature in an orthogonal grid as shown in Figure 6:

- direction X, or transverse direction, contains rows of identical polar units in arch formation (in this case, 5 polar units per arch);

- direction Y, or longitudinal direction, contains parallel rows of identical translational units connecting the polar arches.

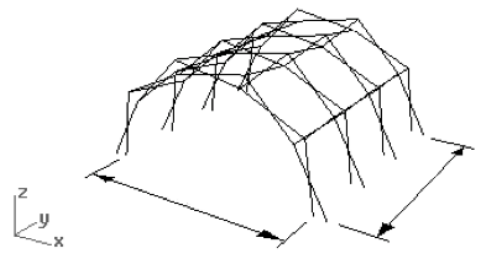

Figure 6: Perspective view of a barrel vault [5].

\subsubsection{Domes}

Besides barrel vaults, also dome geometries are considered in this paper. Domes are not only architecturally and structurally viable structures; they can also serve as a geometric transition to more exotic and interesting shapes thanks to the use of angulated scissor elements.

To minimize distortion of two- or three-way grids over a sphere, polyhedra can be used. A selection is made of polyhedra with an equal edge length, because an identical USC in the whole global structure is the starting point. The selection results in a multitude of different geometries for the investigated architectural dome structures. The following polyhedra are considered: icosahedron, dodecahedron, icosidodecahedron, 'buckyball' (truncated icosahedron) and an adjusted rhombic triacontahedron.

A dome structure, independent from the considered polyhedron, is made deployable by substituting every edge of the polyhedron by scissor elements. For 
this purpose angulated scissors are implemented because of their beneficial properties, such as the ability to develop a more stable deployment process for shapes capable of retracting towards their proper perimeter. Figure 7 shows how the adjusted rhombic triacontahedron forms the basis of a deployable dome using angulated scissors.
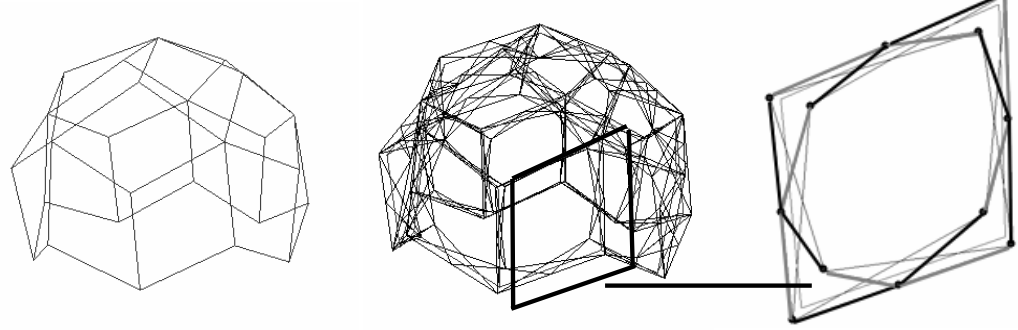

Figure 7: The adjusted rhombic triacontahedron - deployable dome structure with scissor elements - detail of edge replacement by angulated units [5].

\subsection{Geometric dimensions}

A USC will be designed with the ability to configure both barrel vaults as domes. To reach this possibility, the USC must be able to function as the three standard scissor units: translational, polar and angulated, depending on the desired end configuration.

Because hinge displacements have a dramatic influence on the structure shape, in this section decisions will be made concerning the different geometric dimensions based on possible hinge positions. These are the dominating aspect for the geometry: because all the components are identical, i.e. the USC, the only difference between the configurations is the position of the pivot hinge.

\subsubsection{Angulated part of the component}

The angulated element is determined by the different geometric polyhedra to form radially deployable domes. Because of the fixed geometry of the polyhedra, also the angulated part of the USC is relatively fixed. If the length of the kinked beam of an angulated scissor unit is considered as a parameter, the height is determined for a certain polyhedron (Figure 8).

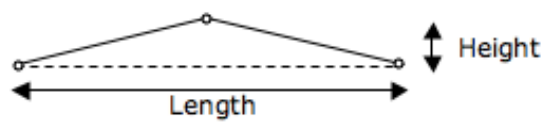

Figure 8: Parameters of an angulated beam. 
A length of $2 \mathrm{~m}$ is chosen because of the resulting range from low to high spans for multifunctional deployable structures. Moreover, a length of $2 \mathrm{~m}$ seems to be still manually manageable. A $2 \mathrm{~m}$ length for the angulated scissor results for each considered polyhedron in a certain height. Further, a selection is made between these different height values. The decision is based on the feasibility of the distance between the intermediate hinge positions, from a manufacturing point of view. For that reason the icosidodecahedron is excluded: the corresponding height value was too close to other values.

Figure 9 presents the ultimate geometric dimensions for the angulated part of the USC for which six different dome structures can be built geometrically.

\begin{tabular}{|c|c|c|c|c|}
\hline & $\begin{array}{l}\text { Hinge position } \\
{[\mathrm{cm}]}\end{array}$ & Dome polyhedron & Elements/edge & $\begin{array}{l}\text { Span } \\
\text { Dome }[\mathrm{m}]\end{array}$ \\
\hline 1 & 10.2 & Buckyball & Double & 16.9 \\
\hline 2 & 16.4 & $\begin{array}{l}\text { Adjusted rhombic } \\
\text { triacontahedron }\end{array}$ & Double & 12.2 \\
\hline 3 & 20.6 & Buckyball & Single & 8.5 \\
\hline 4 & 28.4 & Icosahedron & Double & 6.2 \\
\hline 5 & 33.8 & $\begin{array}{l}\text { Adjusted rhombic } \\
\text { triacontahedron }\end{array}$ & Single & 6.1 \\
\hline 6 & 38.2 & Dodecahedron & Single & 5.2 \\
\hline & 1 & $\begin{array}{rl}0 & 6 \\
0 & 5 \\
0 & 4 \\
0 & 3 \\
0 & 2 \\
0 & 2 \\
\circ & 1 \\
L= & \end{array}$ & & \\
\hline
\end{tabular}

Figure 9: Ultimate geometric dimensions for the angulated part of the USC [5].

\subsubsection{Translational/polar part of the component}

Compared to the relative fixed geometry of the angulated elements in the domes, the polar and translational units allow a bigger freedom in geometry choice.

A polar unit is simply obtained by moving the intermediate hinge of a translational unit away from the middle of the beams. This eccentricity of the revolute joint creates curvature when the units become deployed. A beam from a translational and polar unit can thus simply be combined into one beam with several hinge positions (Figure 10).

The length of the translational or polar beam is fixed on $2 \mathrm{~m}$ as determined in the previous section. The parameters on which can be anticipated are the number of units and the eccentricity of the polar hinges. Configurations are investigated with a number of units from 4 to 16 to obtain a wide range of barrel vault spans and eccentricities are considered with a minimum distances of $5 \mathrm{~cm}$ because of practical reasons. 


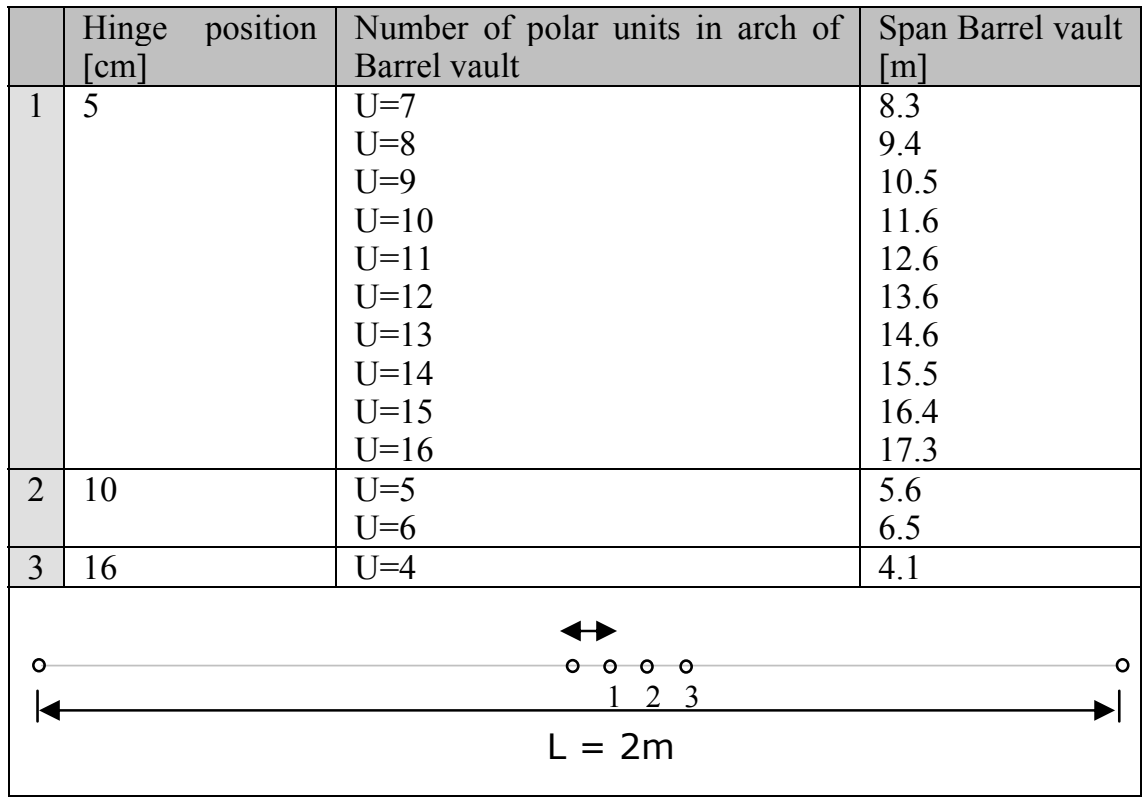

Figure 10: Ultimate geometric dimensions for the translational/polar part of the USC [5].

The result is that only the eccentricities of $5 \mathrm{~cm}, 10 \mathrm{~cm}$ and $16 \mathrm{~cm}$ have to be regarded to form thirteen barrel vaults differing in span and shape (Figure 10).

\subsection{Geometric shape}

In the previous section the geometric dimensions were determined. The positions of the pivot hinges are found with the ability to compose them in such way that nineteen different architectural structures can be configured. The next step is to constitute a shape for the scissor component based on the theoretical positions of the hinges. A shape, easy to manufacture, is formed by simply connecting the hinge positions with beams. Figure 11 illustrates the designed Universal Scissor Component with the different elements.

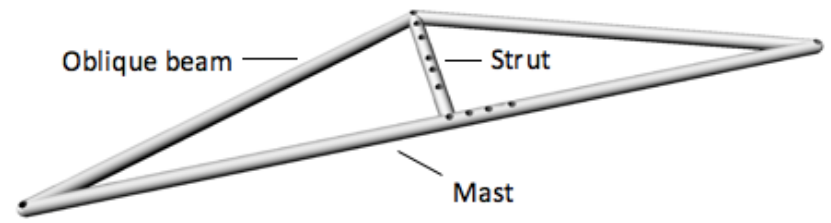

Figure 11: Universal Scissor Component [5]. 


\subsection{Deployment}

Not only do the hinge positions determine the ultimate shape of the deployable structure, they also have an impact on the deployment behaviour which differs between barrel vaults and domes. In the case of the barrel vaults, the deployment has a two dimensional character: the transformation proceeds automatically in the transversal and longitudinal direction, as shown in Figure 12. For the domes, the deployment transforms radially. While deploying the geometric shape stays constant, only a variation in span is noticed (Figure 13).

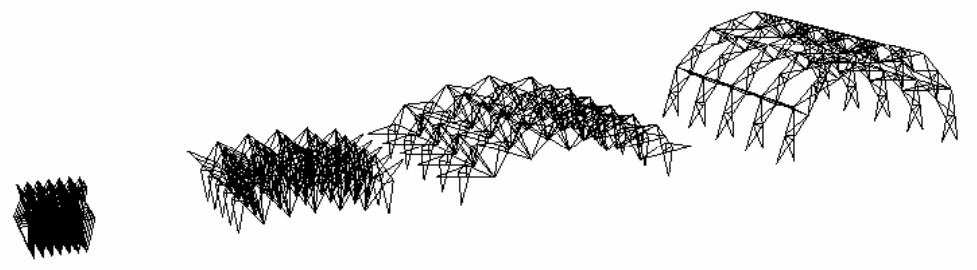

Figure 12: Barrel vault with USCs in four deployment configurations [5].
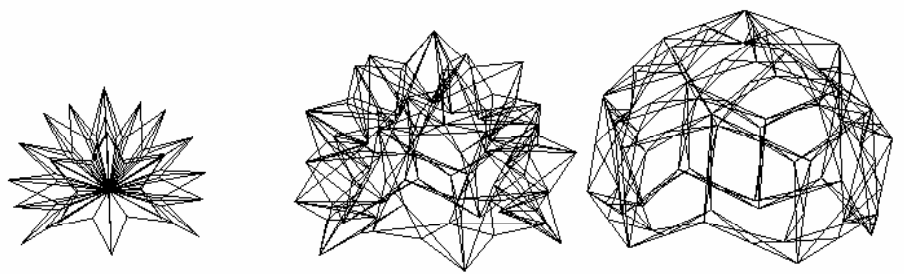

Figure 13: Deployable dome based on the adjusted rhombic triacontahedron from a fully closed to an open configuration [5].

\subsection{Structural evaluation of the USC concept}

The key element with scissor structures is that there is a direct and mutual relationship between the geometry, the kinematics and the structural response of the system. Besides the geometrical properties, inherent to the kinematic deployment behaviour, as explained in the previous sections, a preliminary structural study is conducted to investigate the feasibility of the proposed concept.

As a reference case study, the largest barrel vault $(17 \mathrm{~m} \times 17 \mathrm{~m})$ is analysed structurally in the fully deployed configuration, where the mechanism is blocked and it acts as a load-bearing structure (Figure 14). The structure is calculated using the Eurocode standards. Beside self-weight, also climate loads (transverse and longitudinal wind and snow) are considered as indicated by Figure 15 . 
Relative small cross sections are found (maximum $51 \mathrm{~mm}$ ), compared to the covered area, proving the preliminary feasibility of these types of constructions. But it is to be expected that nearly all of the USC beams are over-dimensioned.

Moreover, in previous research it is stated that traditional scissor elements show a low to medium structural efficiency due to existing bending moments [8]. But this first structural calculation proves that the designed USC rather excludes bending moments, thus increasing the structural efficiency of the structure.

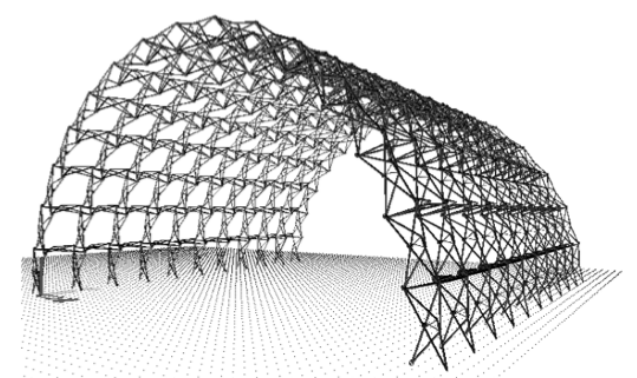

Figure 14: Model of barrel vault in software program SCIA ESA-PT [5].

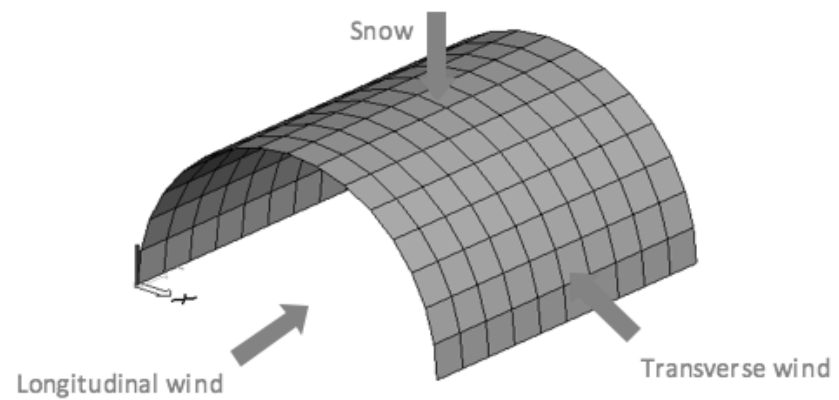

Figure 15: Schematic representation of climate loads [5].

\section{Conclusions}

The aim of the work was to develop an innovative construction system based on a Universal Scissor Component (USC) for deployable structures.

Based on different configurations of structures - barrel vaults and domes decisions were made on the geometric dimensions and the shape of the component. Considering feasible hinge positions, the design process resulted in a USC capable of configuring nineteen different architectural structures with 
specific deployment behaviour (Figure 16). The USC is the unique component in all the investigated configurations; the only difference is the position of the pivot hinge.

A preliminary structural analysis has proven the feasibility of the proposed concept and indicates the increased structural efficiency of scissor structures using USCs.

Further research and a detailed analysis are required to optimise this construction system in terms of weight-per-covered-area-ratio and material- and energy use. Deployable structures have become increasingly popular, but few have been realised successfully. Further work can consist of a better understanding of the design parameters and the related allowable tolerances and imperfections, in order to respect the architectural function of the structure and to guarantee a successful deployment and folding. Their further development calls for research into fundamental issues regarding the shape of the constitutive elements, the connections, the membrane, the deployment behaviour, and the structural performance, both during and after the transformation process. The proposed USC-concept makes reuse and adaptability possible: it is well equipped to meet the demands of a rapidly changing society while embracing the concept of sustainable design.
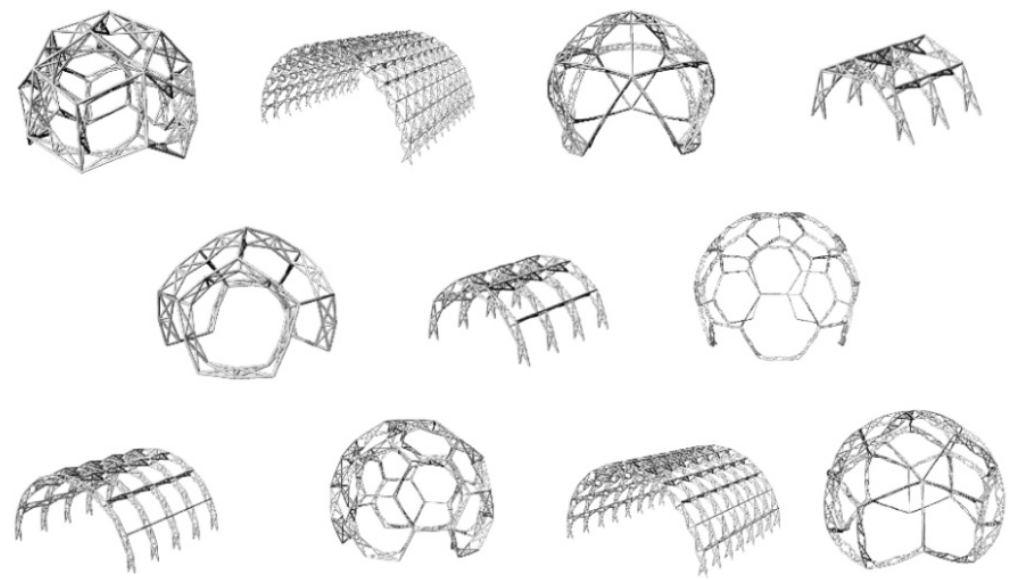

Figure 16: Selection of the possible deployable structures composed of the Universal Scissor Component [9].

\section{References}

[1] Jensen, F.V. Concepts for retractable roof structures. PhD Dissertation University of Cambridge, 2004.

[2] Rückert, G.C. Wandelbare hybride Konstruktionen Von der morphologischen Studie zum Prototyp. PhD Dissertation Technischen Hochschule Zürich, 2000. 
[3] Gantes, C.J., Deployable Structures: Analysis and Design, WIT Press, pp. 189, 2001.

[4] De Temmerman, N. Design and Analysis of Deployable Bar Structures for Mobile Architectural Applications. PhD Dissertation Vrije Universiteit Brussel, 2007.

[5] Alegria Mira, L. Design and Analysis of a Universal Scissor Component for Mobile Architectural Applications. Master's Dissertation Vrije Universiteit Brussel, 2010.

[6] Escrig, F. Expandable space structures, Space Structures Journal, Vol 1, No 2, pp. 79-91, 1985.

[7] Escrig, F. \& Valcarel, J.P. Geometry of Expandable Space Structures. International Journal of Space Structures, Vol 8, Nos 1\&2, pp. 71-84, 1993.

[8] Hanaor, A. \& Levy, R. Evaluations of Deployable Structures for Space Enclosures. International Journal of Space Structures, Vol 16, No 4, pp. 211 229, 2001.181

[9] Alegria Mira, L. \& De Temmerman, N., Design and Analysis of a Universal Scissor Component for Mobile Architectural Applications. Proc. of the Int. Association for Shell and Spatial Structures (IASS) Symposium Shanghai, eds. Q. Zhang, L. Yang \& Y. Hu, China Architecture \& Building Press, pp. 815-826, 2010. 